\title{
The Contribution of NMDA and AMPA Conductances to the Control of Spiking in Neurons of the Deep Cerebellar Nuclei
}

\author{
Volker Gauck ${ }^{1}$ and Dieter Jaeger ${ }^{2}$ \\ ${ }^{1}$ Department of Cognitive Neurology, University of Tuebingen, 72076 Tuebingen, Germany, and ${ }^{2}$ Department of Biology, Emory University, Atlanta, \\ Georgia 30322
}

\begin{abstract}
We performed whole-cell patch-clamp recordings in vitro to investigate the integration of excitatory and inhibitory inputs in neurons of the deep cerebellar nuclei (DCN) by applying synthetic synaptic input patterns with dynamic clamping. We explored an input regime in which excitation and inhibition had an ongoing baseline rate because both input pathways show ongoing activity in vivo. We found that spiking was time-locked to transients in the inputs, consisting of brief decreases in inhibitory or increases in excitatory conductance. Such input transients were caused by synchronization among multiple inputs. However, we found that temporal synchrony in the inhibitory input pathway had preferential access to the control of DCN spiking, because the large NMDA component of the excitatory inputs smoothed out temporal transients in this pathway. Thus, synaptic integration in the DCN appears to be tuned to allow the cerebellar cortical output from Purkinje cells preferential access to the control of DCN spiking. The effect of temporal modulations in the inhibition was further enhanced by the voltage dependence of the NMDA inputs. Thus, the presence of a baseline of mossy and climbing fiber inputs boosted depolarizing responses caused by reduced inhibition by the voltage-dependent increase in inward NMDA current. Overall, our results show that correlated activity or pauses in populations of Purkinje cells are well suited to the dynamic control of DCN spiking. In addition, strong transients in excitation can directly drive DCN responses that bypass cerebellar cortical processing.
\end{abstract}

Key words: NMDA; AMPA; GABA; synaptic integration; cerebellum; coding

\section{Introduction}

Neurons in the deep cerebellar nuclei (DCN) receive massive inhibitory input from the Purkinje cells in the cerebellar cortex, which accounts for $>70 \%$ of all synapses in the DCN (Palkovits et al., 1977; De Zeeuw and Berrebi, 1995). In a previous study we focused on how inhibitory input could control the frequency and accuracy of DCN spiking (Gauck and Jaeger, 2000) using the technique of dynamic clamping in vitro. We found that simulated in vivo Purkinje cell inputs are in fact well suited for the control of spike frequency and timing in DCN neurons. Nevertheless, beyond relaying the output of cerebellar cortical processing to the rest of the brain, the DCN also receive excitatory inputs that are mainly derived from collaterals of the mossy fiber and climbing fiber input systems to cerebellar cortex. These excitatory collaterals could in principle be well suited to drive DCN activity patterns in a direct loop of cerebellar processing that bypasses the cerebellar cortex altogether. It is thus important to gain an understanding of whether the excitatory input conductances that may be expected in vivo are likely to be sufficient to strongly modulate DCN spiking, and how such excitatory control would interact with the inhibition exerted by cerebellar cortex. The excitatory input is made more powerful and possibly dynamically

\footnotetext{
Received March 10, 2003; revised July 7, 2003; accepted July 7, 2003.

This work was supported by a grant from the Federal Ministry of Education and Research (Fö:01KS9602) and the Interdisciplinary Center of Clinical Research Tübingen (to V.G.) and by National Institute of Mental Health Grant R29 MH57256 (to D.J.).

Correspondence should be addressed to Volker Gauck, Department of Cognitive Neurology, University Tuebingen, Auf der Morgenstelle 15, 72076 Tuebingen, Germany. E-mail: volker.gauck@uni-tuebingen.de. Copyright $\odot 2003$ Society for Neuroscience $\quad$ 2270-6474/03/238109-10\$15.00/0
}

more complex by the presence of a large NMDA conductance, which is only weakly voltage-dependent, even in the presence of magnesium (Audinat et al., 1992; Anchisi et al., 2001). Furthermore, two NMDA components with different kinetics and different voltage dependencies were described (Cull-Candy et al., 1998; Anchisi et al., 2001). Although the decay time constants of the NMDA current are faster compared with many other cell types, they are still much longer than the AMPA component also present. Based on these studies, the total charge carried by NMDA current should far outweigh AMPA currents in DCN neurons. It is thus important to understand how the specific kinetic properties and voltage dependence of NMDA conductance in DCN neurons may be influencing the process of synaptic integration and the ensuing control of spiking.

To examine excitatory input processing in DCN neurons, we performed a dynamic clamp study in vitro analogous to our previous one focusing only on inhibition (Gauck and Jaeger, 2000). In vivo, DCN neurons receive ongoing input from Purkinje cells, mossy fibers, and climbing fibers (Eccles et al., 1971; Cazin et al., 1980; Savio and Tempia, 1985; Stratton et al., 1988; van Kan et al., 1993; Gamlin and Clarke, 1995; Matsuzaki and Kyuhou, 1997). Therefore, we simulated input conditions that mimicked an ongoing balance of excitatory and inhibitory inputs. We found that the spiking of DCN neurons could be determined by excitation as well as by inhibition depending on the degree of synchronization within the respective input pathway. The presence of a large NMDA conductance allowed inhibition to dominate in the control of spike timing for most input patterns. Furthermore, the impact of fluctuations in inhibition was enhanced by the voltage dependence of the slow NMDA component. 


\section{Materials and Methods}

Electrophysiology. All animal procedures used in these studies fully complied with the National Institutes of Health guidelines on animal care and use. DCN neurons were recorded in sagittal cerebellar slices $(250 \mu \mathrm{m})$ of 10 - to 18 -d-old male Sprague Dawley rats in whole-cell patch-clamp mode. We were unsuccessful in recording from older animals, because fibers in the DCN become very heavily myelinated beyond this age, which diminishes both visibility and viability of neurons after slicing. No differences were found in the morphology of DCN neurons from 8- or 20-d-old rats (Sultan et al., 2003), suggesting that this structure matures early. We also found no differences in response properties for neurons recorded between 10 and $18 \mathrm{~d}$ of age. Electrodes had resistances between 4 and $7 \mathrm{M} \Omega$ and were filled with (in $\mathrm{mM}$ ): K-gluconate 140, HEPES 10, $\mathrm{NaCl} 10$, EGTA 0.2, MgATP 4, NaGTP 0.4, and glutathione 5. The extracellular solution used for recording contained (in mM): $\mathrm{NaCl} 124, \mathrm{KCl} 3$, $\mathrm{KH}_{2} \mathrm{PO}_{4} 1.2, \mathrm{NaHCO}_{3} 26, \mathrm{CaCl}_{2} 2, \mathrm{MgSO}_{4} 1.9$, and glucose 20. Inhibitory synaptic inputs were blocked either with $40 \mu \mathrm{m}$ bicuculline methiodide or with $10 \mu \mathrm{m}$ picrotoxin. Excitatory synaptic inputs were blocked with $100 \mu \mathrm{M}$ kynurenic acid. All chemicals were obtained from (Sigma, Deisenhofen, Germany). Neurons in the DCN were selected visually to obtain recordings only from cells with a soma diameter exceeding $15 \mu \mathrm{m}$, which has been described as a defining characteristic of excitatory projection neurons (Batini et al., 1992). The recording temperature was $32^{\circ} \mathrm{C}$ (i.e., between room temperature and physiological temperature) to achieve a viable compromise between slow kinetics at low temperatures and fast tissue degradation at $37^{\circ} \mathrm{C}$ when recording in vitro. We cannot exclude that at $32^{\circ} \mathrm{C}$ the excitability of the recorded cells was below that under in vivo conditions because of slowed channel kinetics. Significant differences in synaptic processing between these temperatures have not been reported, however.

Dynamic current clamping. We used the technique of dynamic current clamping (Robinson and Kawai, 1993; Sharp et al., 1993) to inject synthetic synaptic input into DCN neurons in vitro according to Equation 1: $I_{\text {inj }}=G_{\mathrm{ex}}(t) *\left(V_{\mathrm{m}}-E_{\mathrm{ex}}\right)+G_{\mathrm{in}}(t) *\left(V_{\mathrm{m}}-E_{\mathrm{in}}\right)$. The injected current $\left(I_{\text {inj }}\right)$ was updated on-line during the recording for each sampling point at a rate of $10 \mathrm{kHz}$. To achieve this sampling rate with a temporal precision in the microsecond range, we used a custom-made C-routine (S. Melon and V. Gauck, unpublished software) running under the RTLinux operating system. The computer board used for data acquisition was a PCI-6052E (National Instruments). The simulated excitatory $\left[G_{\mathrm{ex}}(t)\right]$ and inhibitory $\left[\left(G_{\mathrm{in}}(t)\right]\right.$ synaptic conductance traces were calculated off-line before the recording and stored on hard disk. They represent the temporal sum over all excitatory $\left[G_{\mathrm{ex}}(t)=\Sigma g_{\mathrm{ex}}\right]$ and all inhibitory $\left[G_{\text {in }}(t)=\Sigma g_{\text {in }}\right]$ input trains. The unitary conductances $g_{\text {ex }}$ and $g_{\text {in }}$ (Fig. 1) were calculated as dual-exponential functions according to Equation 2: $g_{\text {unitary }}=1 /\left(\tau_{\text {decay }}-\tau_{\text {rise }}\right)^{\star}\left(e^{-t / \tau \text { decay }}-e^{-t / \tau \text { rise }}\right)$. Before starting a recording, $G_{\text {ex }}$ and $G_{\text {in }}$ traces were loaded into memory, and for each sampling time, $t_{\mathrm{i}}$, the appropriate values $G_{\text {ex }}\left(t_{\mathrm{i}}\right)$ and $G_{\text {in }}\left(t_{\mathrm{i}}\right)$ were used together with the recorded momentary membrane potential $V_{\mathrm{m}}\left(t_{\mathrm{i}}\right)$ to update $I_{\text {inj }}\left(t_{\mathrm{i}}\right)$ (Eq. 1). The reversal potentials for excitatory $\left(E_{\mathrm{ex}}\right)$ and inhibitory input $\left(E_{\text {in }}\right)$ were 0 and $-70 \mathrm{mV}$, respectively.

The inhibitory input sequences from putative in vivo Purkinje cell activity were constructed as in our preceding study (Gauck and Jaeger, 2000). Unitary conductances had a rise time of $0.93 \mathrm{msec}$ and a decay time of 13.6 msec (Anchisi et al., 1998). A set of 400 such GABA $_{\mathrm{A}}$-type input elements were activated randomly at a mean firing rate of $35 \mathrm{~Hz}$, and the conductances were summed to generate $G_{\text {in }}$. We restricted the present analysis to the unitary conductance amplitude of $69 \mathrm{pS}$ for inhibitory inputs, which is identical to the gain factor 16 in our previous study, i.e., resulting in an average mean level of $16 \mathrm{nS}$ of inhibitory conductance. The 400 input elements were either activated in 100 independently spiking groups or in only 10 independently active input groups, duplicating our previously used conditions of intermediate and high input synchronization in the inhibitory pathway (Gauck and Jaeger, 2000). We discarded our previously used additional conditions of 400 independent input groups and unitary amplitudes of $<69 \mathrm{pS}$, because we found these to result in regular or slow firing DCN patterns uncharacteristic of in vivo recordings. Purkinje cell synapses in the DCN are likely to be significantly
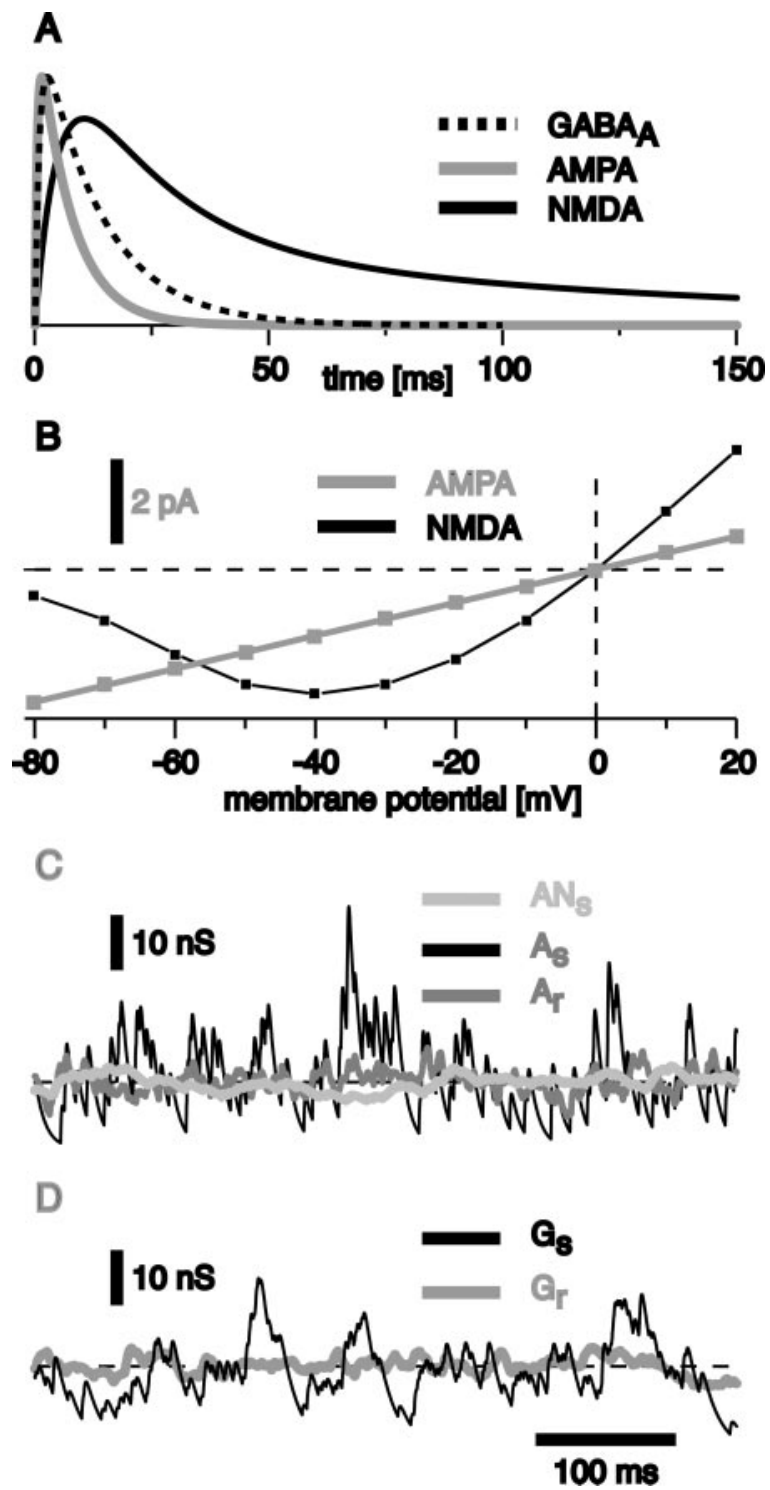

Figure 1. Stimulus properties. $A$, Unitary conductance traces for AMPA (gray), NMDA (black), and $G_{A B A_{A}}$ (dotted black) inputs. The GABA $A_{A}$ amplitude (peak of $69 \mathrm{pS}$ ) is scaled down to the AMPA peak conductance ( $39 \mathrm{pS})$ to allow for a better comparison of the time course. $B$, Currentvoltage plot of the AMPA (gray) and NMDA peak currents (black). Note that the NMDA current is $83 \%$ of AMPA at $-60 \mathrm{mV}$ but is more than double the AMPA current positive to $-30 \mathrm{mV}$. C, A 0.5 sec sample segment of the excitatory conductance traces used in the experiments depicted in Figures 3-7. The traces correspond to synchronized AMPA input $\left(A_{S}\right.$, black), random AMPA input $\left(A_{R}\right.$, dark gray), and synchronized, mixed AMPA-NMDA input ( $A N_{S}$, light gray). The AMPA-NMDA trace shown here is given for a constant membrane potential of $-40 \mathrm{mV} . D, A 0.5 \mathrm{sec}$ sample segment for inhibitory $G_{A B A_{A}}$-type input for synchronized $\left(G_{S}\right.$, black) and random $\left(G_{R}\right.$, gray) input conditions. In this and the following figures, the line thickness was increased for lines with lighter gray values to improve their visibility.

depressed because of short-term plasticity when the ongoing input rate is high (Telgkamp and Raman, 2002; Pedroarena and Schwarz, 2003), however, the synaptic amplitudes we use are still at the low end of the spectrum of synaptic amplitudes shown in the same studies. We did not include short-term plasticity in the present study because we limited our analysis to ongoing fast input activity characteristic of Purkinje cell firing in vivo, which would lead to a near constant level of such depression. The present study also excluded the examination of transient increases and decreases in input activity because the parameter space of such possible changes is nearly unlimited and is beyond the scope of the basic interactions between excitation and inhibition examined here.

In our previous study we found that a constant baseline of excitation of 
$12 \mathrm{nS}$ conductance was required in conjunction with our applied inhibitory inputs so that realistic spike frequencies could be achieved. In the present study we replaced the time invariant excitation with realistic patterns of excitatory input, however, we kept a mean level of $12 \mathrm{nS}$ of excitatory conductance for all stimuli used. By keeping the mean conductance level constant, we could exclude that shifts in the balance of excitation and inhibition interfered with our determination of AMPA and NMDA conductance effects on spike control. The main effect of shifting the balance of excitation and inhibition was examined in our previous study (Gauck and Jaeger, 2000), and consisted of a change in output spike rate without altering the effects of input synchronicity and total conductance amplitude on output spiking. Realistic excitatory input to DCN neurons is composed of an AMPA component, a fast NMDA component, and a slow NMDA component (Anchisi et al., 2001), and is provided by collaterals of mossy fibers and climbing fibers that also innervate cerebellar cortex (Sugihara et al., 1999; Wu et al., 1999; Shinoda et al., 2000). The total number of excitatory synapses on DCN neurons is $\sim 2000$ (Palkovits et al., 1977) and represents an upper estimate for the number of mossy and climbing fibers converging on a single neuron because each input fiber may make multiple contacts on a DCN neuron. We used 100 excitatory inputs, which corresponds to a convergence factor of 20 synapses per incoming axon. No experimental data exist on this convergence factor, but we found that a larger number of independently firing inputs approximates the condition of a time-invariant conductance. The mean activation rate of excitatory inputs was set to $20 \mathrm{~Hz}$ in accordance with in vivo recordings (Eccles et al., 1972; Cazin et al., 1980; van Kan et al., 1993; Gamlin and Clarke, 1995; Matsuzaki and Kyuhou, 1997). No difference between mossy and climbing fiber inputs has been described in the DCN, and we simulate only one homogenous group of excitatory inputs. The AMPA, fast NMDA, and slow NMDA components of each excitatory input were calculated according to Equation 3: $G_{\text {ex }}(t)=\left[G_{\text {AMPA }}(t)+G_{\text {fastNMDA }}(t) \cdot f_{\text {fast }}\left(V_{\mathrm{m}}\right)+G_{\text {slowNMDA }}(t)\right.$ - $\left.f_{\text {slow }}\left(V_{\mathrm{m}}\right)\right]$. The voltage dependencies of the NMDA components (Fig. $1 B$ ) were simulated by multiplication of the corresponding conductance traces with the factor $f$, described by Equation 4: $f\left(V_{\mathrm{m}}\right)=1 /[1+$ $\left.P_{1}{ }^{*} \exp \left(-\mathrm{P}_{2}{ }^{*} V_{\mathrm{m}}\right)\right]$. As described above for $G_{\mathrm{ex}}$ and $G_{\mathrm{in}}$, this factor was updated on-line during recording at a rate of $10 \mathrm{kHz}$. The parameters of AMPA and NMDA inputs were set to match the data of Anchisi et al. (2001). The best fit to the reported voltage dependencies of the NMDA current (Anchisi et al., 2001) was achieved by setting the parameters P1 and P2 to 0.002 and 0.109 for the fast NMDA component and to 0.25 and 0.057 for the slow NMDA component. The rise and decay time constants were 0.5 and $7.1 \mathrm{msec}$ for AMPA, 5 and $20.2 \mathrm{msec}$ for fast NMDA, and 5 and $136.4 \mathrm{msec}$ for slow NMDA (Fig. $1 A$ ). The fast unitary NMDA conductance had a peak value of $57 \%$ of the AMPA peak, and the slow unitary NMDA conductance had a peak value of $28 \%$ of the AMPA peak at $-60 \mathrm{mV}$ (Fig. $1 \mathrm{~A}$ ). Thus, the sum of the fast and slow unitary NMDA conductances had a peak value of $83.2 \%$ that of the AMPA peak at -60 $\mathrm{mV}$ (Fig. 1A). The unitary peak conductance amplitude of the AMPA conductance was set to $39 \mathrm{pS}$ to generate the desired $12 \mathrm{nS}$ average excitatory conductance. In the presence of voltage-dependent NMDA conductance, the actual average excitatory conductance is a function of the membrane potential trajectory. Thus, to compute a stimulus with an expected average excitatory conductance of $12 \mathrm{nS}$, we used $-40 \mathrm{mV}$ as the assumed membrane potential, which was the average combined reversal potential of excitation and inhibition of our stimuli when the NMDA conductance was voltage-independent. We previously showed that the actual mean $V_{\mathrm{m}}$ is very close to this average combined reversal potential because of the shunting effects of ongoing input conductances (Gauck and Jaeger, 2000). The time course of unitary excitatory and inhibitory input conductances was taken from experimental data obtained at a temperature of $25^{\circ} \mathrm{C}$ (Anchisi et al., 1998, 2001) and might therefore underestimate their speed at physiological temperatures in vivo. However, elevated temperature will shorten the kinetics of excitatory and inhibitory inputs by a similar factor, and therefore the relative synchrony of the input pathways will not be affected.

In our previous study we found that input synchronization has a large effect on spike rate and spike timing (Gauck and Jaeger, 2000). Therefore we focused the present investigation on how varying amounts of input synchronization in the excitatory and inhibitory input pathways would determine the output spike pattern. Furthermore, we aimed at isolating the contribution of AMPA and NMDA conductances in the effect of excitation on spiking. To achieve these distinctions we constructed stimuli, which included either random or synchronized excitatory or inhibitory inputs. To isolate the effect of temporal information in either the excitatory or inhibitory input pathway alone, an additional condition was introduced, in which the other pathway was replaced with a time invariant input conductance. For random input patterns we activated all 100 inputs (inhibition or excitation) independently with the specified mean rates. For a synchronized input, we coupled groups of 10 inputs to the same time series of presynaptic activation. To examine the contribution of NMDA conductances to output spiking, we constructed stimuli with the same time series of activation but a complete replacement of NMDA conductance with a larger unitary AMPA conductance (691 pS). The role of the voltage dependence of the NMDA conductances was further dissected by comparing the voltage-dependent form described above with a voltage-independent form, in which a constant $V_{\mathrm{m}}$ of -40 $\mathrm{mV}$ was used to update the factors $f_{\text {fast }}\left(V_{\mathrm{m}}\right)$ and $f_{\text {slow }}\left(V_{\mathrm{m}}\right)$ (Eq. 4$)$, resulting in an average total excitatory conductance of $12 \mathrm{nS}$ (see above). By this scaling procedure, the average total $\left(G_{\text {ex,av }}\right)$ and the unitary peak $\left(g_{\text {ex,peak }}\right)$ amplitudes of the voltage-dependent and the voltageindependent excitatory inputs were set to identical values for the reference potential of $-40 \mathrm{mV}$. Consequently, the voltage-dependent input $\left(G_{\text {ex,av }}\right.$ or $\left.g_{\text {ex,peak }}\right)$ was smaller than its voltage-independent counterpart for voltages less than $-40 \mathrm{mV}$, whereas it was larger for voltages more than $-40 \mathrm{mV}$. The reference potential of $-40 \mathrm{mV}$ was chosen because it is very close to the average membrane potential during the presentation of our stimuli.

Unfortunately the total input conductances impinging onto DCN neurons in vivo have not been determined experimentally. Our simulated input patterns are more likely to underestimate than to overestimate total conductance levels, because only 100 inputs of excitation and inhibition were used, which is below the numbers of synapses found in anatomically estimates (Palkovits et al., 1977). In addition, our unitary conductance amplitudes were also at the low end of expected values estimates (Telgkamp and Raman, 2002; Pedroarena and Schwarz, 2003). On the other hand, we did not simulate synaptic failures, dendritic attenuation, or short-term depression. For these reasons we cannot claim to construct fully natural input patterns, for which data simply do not exist. None of our analyses, however, are dependent on specific choices of absolute input levels or temporal input patterns. Our input conditions chosen should instead be considered as a general case of continuous fluctuating input conductances (see Discussion).

Data analysis. All dynamic current-clamp stimuli had a duration of 10 sec. To exclude onset transients of the stimulation, the first $0.5 \mathrm{sec}$ were excluded from data analysis. Different stimuli were presented in a pseudorandom sequence, which was repeated 2-6 times. Two successive stimuli were separated by a pause of $1 \mathrm{sec}$ during which no current was injected. The evaluated parameters were the mean spike frequency and the mean spike timing precision, following our previous study (Gauck and Jaeger, 2000). To evaluate the spike timing precision, a time window of $\pm 5 \mathrm{msec}$ was defined for each spike, and it was determined how many spikes elicited by repeated stimulus presentations fell into this time window. Averaging this value over all spikes and normalizing it by the number of stimulus repetitions resulted in the percentage of spikes accurately controlled by the input within a time window of $\pm 5 \mathrm{msec}$. The chance level of such coincident firing across trials was calculated by using the same algorithm after shuffling the interspike intervals within a trial, and the overall level of stimulus-controlled precise firing was computed by subtracting this expected chance level. To separate the influence of temporal transients in the excitatory and inhibitory input pathways, we compared spike rates and spike precision values for stimuli, in which either excitatory or inhibitory input conductance were held constant or were modulated at the same time (Fig. 2).

\section{Results}

The presented data were recorded from 25 DCN neurons with soma sizes ranging from 15 to $30 \mu \mathrm{m}$. The soma sizes indicate 


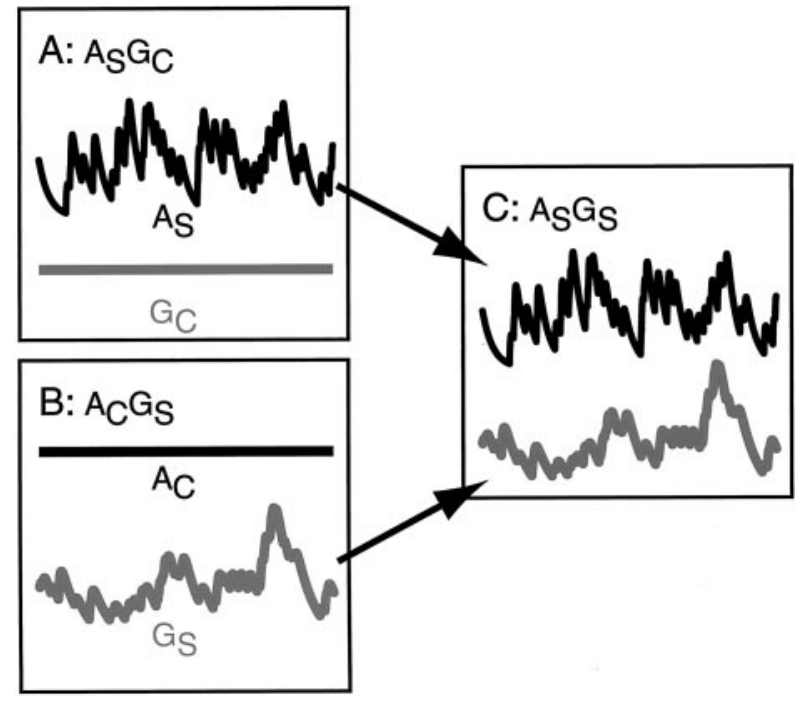

Figure 2. Stimulus design of the experiments presented in Figures 3-6. To separate the influences of excitatory versus inhibitory inputs on the spiking activity of DCN neurons, three stimuli were required in each experiment. $A$, To isolate the influence of temporal fluctuations caused by synchronously active excitatory input elements, a constant inhibitory conductance

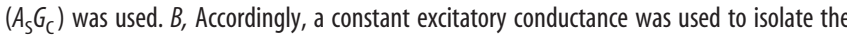
influence of synchronously active inhibitory inputs $\left(A_{C} G_{S}\right)$. C, In a third stimulus, the excitatory and inhibitory conductance traces were both amplitude-modulated according to the activity of the corresponding input pathway $\left(A_{s} G_{s}\right)$. By comparing the resulting spiking pattern of this stimulus to the responses resulting from $A$ and $B$, we could separate the influence of each input pathway on spiking even in the mixed input condition.

that the recorded neurons belong to the group of excitatory projection neurons (Batini et al., 1992). Their spontaneous firing frequency ranged from 0 to $8 \mathrm{~Hz}$.

\section{The control of spiking by a mixed baseline of excitatory and inhibitory inputs}

During in vivo activity, DCN neurons will receive ongoing inputs from excitatory collaterals of mossy and climbing fibers and from inhibitory Purkinje cell synapses. We have previously determined using in vitro dynamic current clamping that an ongoing balance of inhibition and excitation is needed to drive DCN neurons at realistic spike frequencies (Gauck and Jaeger, 2000). Here we ask the question of how the temporal modulation of excitatory and inhibitory input is likely to contribute to the control of output spiking.

In a first stimulus set, we used synchronized excitatory AMPA and NMDA $\left(A N_{s}\right)$ and synchronized inhibitory $\mathrm{GABA}_{\mathrm{A}}\left(G_{\mathrm{s}}\right)$ inputs, each consisting of 10 groups of coactivated sets of synapses (see Materials and Methods). To isolate the contribution of temporal information in each pathway we added stimuli, in which either excitation or inhibition was time-invariant, i.e., constant $\left(A N_{\mathrm{c}}\right.$ or $\left.G_{\mathrm{c}}\right)$ (Fig. 2). The spike response of a typical cell for a short time segment of these stimuli is shown in Figure 3A. The spike rasters show that spike timing was highly conserved for repeated stimulus presentations within each input pattern, indicating that temporal modulation in either inhibition or excitation can control spiking precisely. When both excitation and inhibition contain an identical level of temporal modulation (i.e., 10 sets of synchronized synapses), the spike pattern much resembles that of inhibitory input alone. Thus, time-variant inhibitory input dominates over time-variant excitatory input under these conditions. This result is quantified in Figure $3 C$ for a set of 25 recorded DCN neurons. The data of the sample neuron shown in Figure $3 A$ are
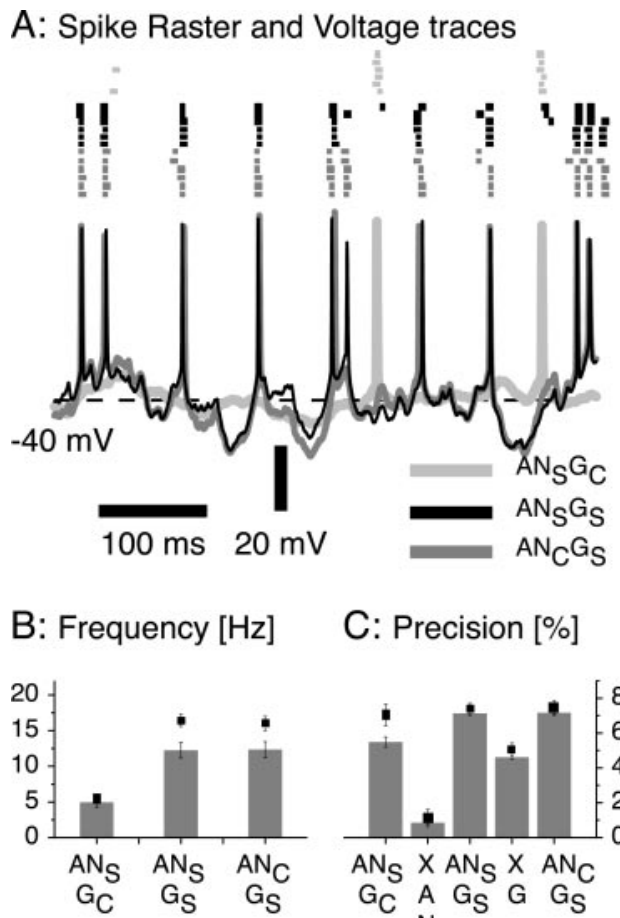

C: Precision [\%]
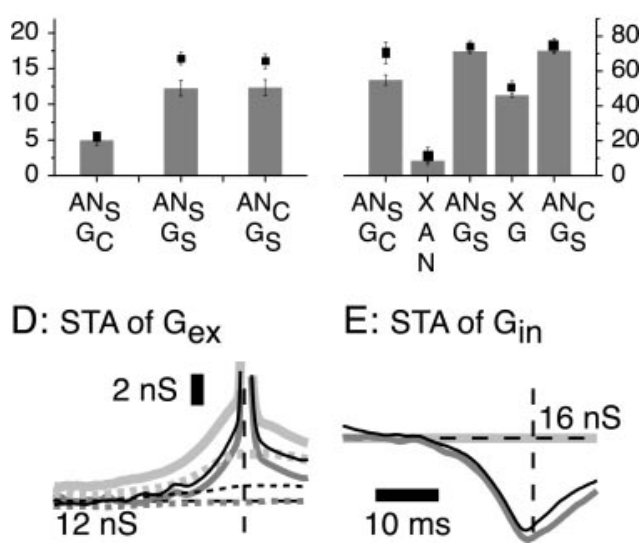

$E$ : STA of $G_{\text {in }}$

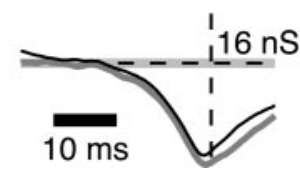

Figure 3. Control of DCN spiking by a combination of synchronized AMPA-NMDA input $\left(A N_{S}\right)$ and synchronized $G A B A_{A}$ input $\left(G_{S}\right)$. To isolate the effect of each pathway, $A N_{S} G_{C}$ combines synchronized excitatory AMPA-NMDA input with constant inhibitory input (light gray). $A N_{C} G_{S}$ combines exclusively voltage-modulated AMPA-NMDA input with synchronized inhibitory $G_{A B A_{A}}$ input (dark gray). $A N_{S} G_{S}$ combines synchronized AMPA-NMDA input with synchronized $G_{A B A_{A}}$ input (black). $A$, Voltage response and corresponding spike raster plots to six stimulus presentations from a typical DCN neuron. $B$, Spike rate averaged over 25 DCN neurons (bars, mean $\pm \mathrm{SE}$ ) and spike rate averaged over six stimulus repetitions from the individual DCN neuron shown in $A$ (squares, mean \pm SD). C, Spike timing precision averaged over 25 DCN neurons (bars, mean $\pm \mathrm{SE}$ ) and spike timing precision averaged over six stimulus repetitions from the sample neuron (squares, mean $\pm S D$ ). The precision for repetitions of the same stimulus condition $\left(A N_{S} G_{C}, A N_{S} G_{S}, A N_{C} G_{S}\right)$ as well as stimuli identical only for excitation (XAN) or inhibition (XG) are shown (see Results). D, Spike-triggered averages of the excitatory conductance for all three stimuli. The solid lines were calculated from the $G_{\text {ex }}$ with voltagedependent NMDA component, whereas the dotted lines were calculated from voltageindependent but otherwise identical versions of $G_{\text {ex }}$ that were created, assuming a constant membrane potential of $-40 \mathrm{mV}$ (see Eq. 4). E, Spike-triggered averages of the inhibitory conductance for all three stimuli. The data in $D$ and $E$ are from the same neuron as in $A$.

also indicated for comparison (black boxes). The frequency of spiking when only excitation is time-variant is much reduced compared with the other input conditions (Fig. 3B), although the average input conductances $G_{\mathrm{ex}}$ and $G_{\text {in }}$ are identical. In addition, the precise spike timing was primarily controlled by fluctuations in inhibition and not excitation, because all input conditions sharing synchronous inhibition resulted in similar spike timing regardless of the presence or absence of synchronous excitation. In Figure $3 C$ and the following figures we identify the contribution of inhibition and excitation to the control of spike timing by analyzing spike timing across repeated stimulus presentations that are either fully identical (e.g., $A N_{s} G_{s}$ ) or are identical only in excitation (e.g., $A N_{s} G_{s}$ vs $A N_{s} G_{c}$ denoted by XAN) or inhibition 
( $A N_{\mathrm{s}} G_{\mathrm{s}}$ vs $A N_{\mathrm{c}} G_{\mathrm{s}}$ denoted by XG). This analysis shows that the spike timing across the $A N_{\mathrm{s}} G_{\mathrm{s}}$ and $A N_{\mathrm{c}} G_{\mathrm{s}}$ conditions with different excitation but identical inhibition (i.e., XG) was mostly identical (Fig. 3C). To further analyze the input events responsible for spike generation the spike triggered averages (STA) of the excitatory and inhibitory conductances $\left(G_{\text {ex }}, G_{\text {in }}\right)$ were calculated (Fig. $3 D, E)$. The STA of $G_{\text {ex }}$ started to rise $\sim 20$ msec before the peak of an action potential when $G_{\text {in }}$ was constant (Fig. 3D,E, solid light gray). When both input streams had synchronized input patterns, the STA of $G_{\text {ex }}$ had a shorter time course and was reduced in amplitude (Fig. 3D, solid black). The STA of $G_{\text {ex }}$ was actually very similar to this shape when excitation was exclusively voltagemodulated (Fig. 3D, solid dark gray). In this case, the modulation of $G_{\mathrm{ex}}$ before spiking is entirely attributable to the voltagedependent increase of $G_{\mathrm{NMDA}}$ caused by membrane depolarization preceding a spike. A voltage-independent form of $G_{\text {ex }}$ was computed for the same excitatory input patterns by assuming a constant feedback membrane potential of $-40 \mathrm{mV}$. The STA of this voltage-independent $G_{\text {ex }}$ was flat for a constant excitatory conductance, of course (Fig. 3D, dashed dark gray). It was also nearly flat when input modulation was present for both excitation and inhibition (Fig. 3D, dashed black), indicating that in this condition excitation contributes little to the control of spike timing. The STA of inhibitory inputs confirms the dominance of inhibition over excitation, because the average decrease of inhibitory input before spiking is near-identical in the presence or absence of excitatory input modulation (Fig. 3E). The shape of the STA of $G_{\text {in }}$ indicates that a decrease of inhibitory inputs in a short time window of 10-20 msec reliably triggers spikes, as previously documented (Gauck and Jaeger, 2000).

\section{The role of input synchronization in controlling output spiking}

To examine whether the dominance of inhibition in the control of DCN spiking was dependent on the amount of synchronization present in the inhibitory pathway, we constructed a second stimulus set, in which all inhibitory inputs were firing randomly $\left(G_{\mathrm{r}}\right)$. All other stimulus properties remained identical to the conditions described above. In response to this reduction in temporal modulation of inhibition pathways, synchronized excitation was now able to dominate in the control of spiking (Fig. 4). Again, the precision of spike timing was high in response to all stimuli, but now the spike patterns across conditions were very similar when the excitation remained highly modulated but inhibition differed (Fig. 4C, XAN). In contrast, identical random inhibition but different excitatory input led to completely different spike patterns (Fig. 4C, XG). Furthermore, now the spike frequency was low for all stimulus conditions (Fig. $4 \mathrm{~B}$ ), indicating that they contained few temporal fluctuations capable of triggering spikes (Fig. 1C,D). The STAs of $G_{\text {ex }}$ were almost identical for the stimuli that had synchronized excitation in common (Fig. $4 D: A N_{\mathrm{s}} G_{\mathrm{c}}=$ light gray, $A N_{\mathrm{s}} G_{\mathrm{r}}=$ black). In contrast, the amplitude of the STA of $G_{\mathrm{r}}$ was substantially lowered in the presence of synchronized excitation (Fig. $4 E: A N_{\mathrm{s}} G_{\mathrm{r}}=$ black, $A N_{\mathrm{c}} G_{\mathrm{r}}=$ dark gray). Nevertheless, it should be noted that random inhibition still made a significant contribution to spike timing in the presence of synchronous excitation (Fig. 4), whereas synchronized excitation had almost no influence in the presence of synchronized inhibition (Fig. 3).

Overall, these data show how the relative influence that excitatory versus inhibitory inputs exerted on spike initiation depends on their synchronization level in DCN neurons. Although the level of synchronization was held constant in our experiments

\section{A: Spike Raster and Voltage traces}
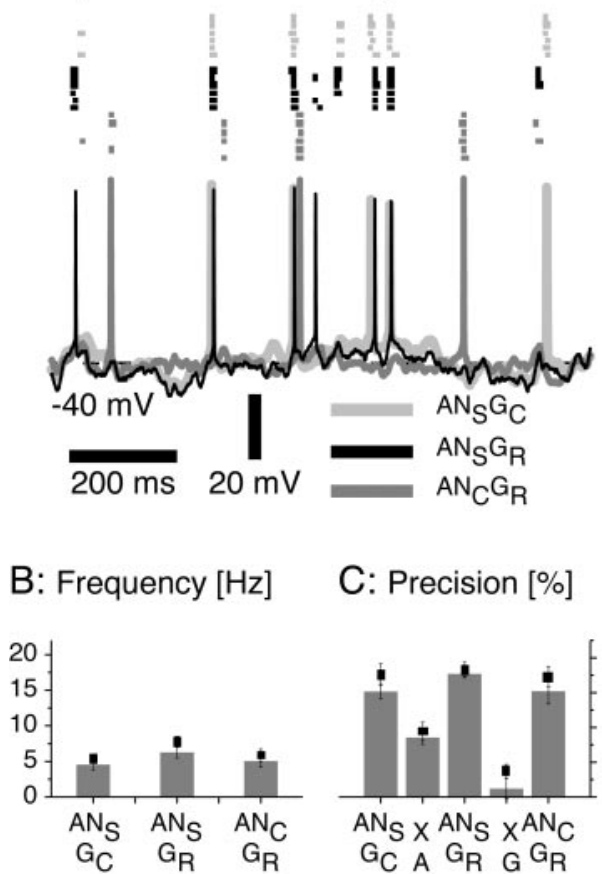

C: Precision [\%]
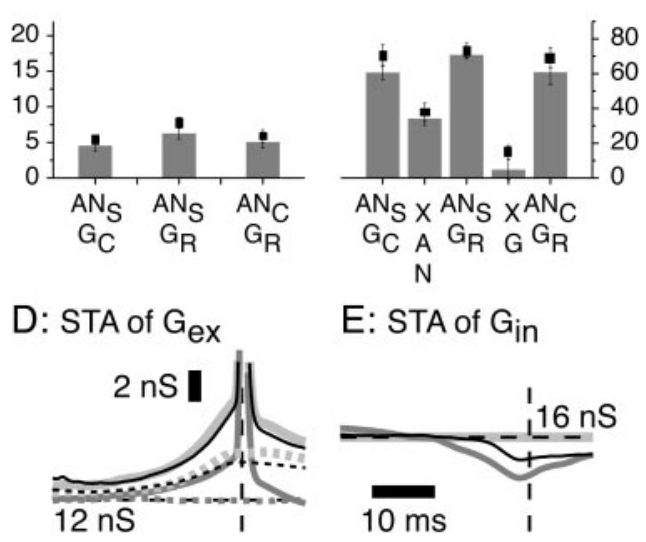

$E:$ STA of $G_{\text {in }}$

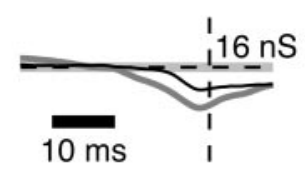

Figure 4. Control of DCN spiking by a combination of synchronized AMPA-NMDA input and random $G_{A B A_{A}}$ inputs. $A N_{S} G_{C}$ combines synchronized excitatory AMPA-NMDA input with constant inhibitory input (light gray). $A N_{C} G_{R}$ combines exclusively voltage-modulated excitatory input with random inhibitory $G A B A_{A}$-type input (black). $A N_{S} G_{R}$ combines synchronized AMPANMDA input with random $G_{A B A_{A}}$ input (dark gray). $A$, Voltage response and corresponding spike raster plots to six stimulus presentations from a typical recording. $B-E$, Same analyses as shown in Figure 3, but for the stimulus set including random instead of synchronized inhibitory inputs ( $n=10$ neurons recorded).

within a particular stimulus, the synchronization could change on short time scales in vivo, whereby the control on spike generation could be shifted from inhibitory to excitatory inputs or vice versa. With respect to synaptic integration in DCN neurons, however, it might be of particular interest that the predominant influence on spike generation was exerted by inhibition for identical synchronization levels of excitation and inhibition, which is likely to allow the cerebellar cortex to exert a privileged level of control over DCN spiking.

\section{The role of the NMDA conductance in synaptic integration}

One possibility that synaptic integration in the DCN favors inhibition is given by the large component of NMDA conductance, which, because of its slow time course, tends to smooth out the effect of temporal modulation in the excitatory input (Harsch and Robinson, 2000). To determine the contribution of this effect to the observed results, we constructed additional stimulus sets, in which the NMDA component of excitatory input conductance was replaced by increasing the unitary AMPA conductance to 691 pS. This increase resulted in maintaining the same average amplitude of $G_{\text {ex }}$ of $12 \mathrm{nS}$ for all stimulus patterns. 

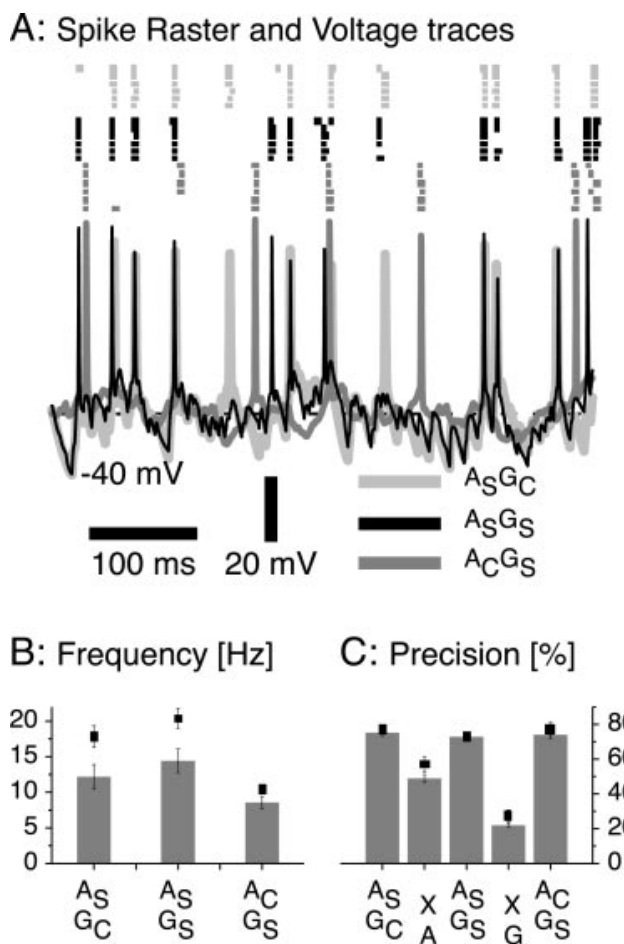

C: Precision [\%]
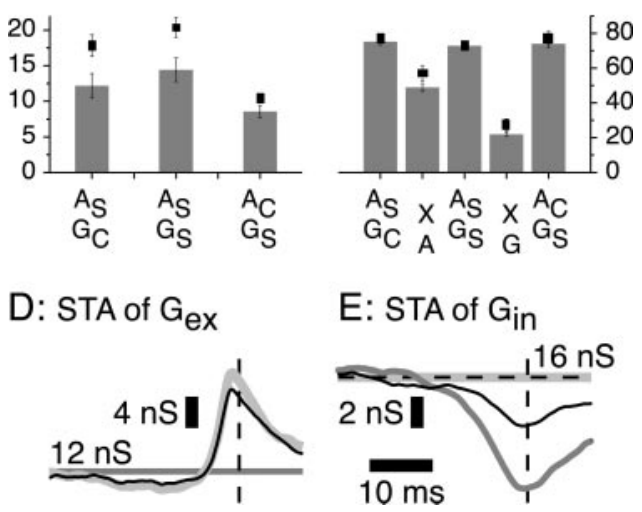

Figure 5. Control of DCN spiking by a combination of synchronized AMPA input (NMDA component replaced by increasing AMPA peak conductance) and synchronized GABA inputs. $A_{S} G_{C}$ combines synchronized excitatory AMPA input with constant inhibitory input (light gray). $A_{C} G_{S}$ combines constant excitatory input with synchronized inhibitory $G A B A_{A}$ type input (dark gray). $A_{S} G_{S}$ combines synchronized AMPA input with synchronized $G A B A_{A}$ input (black). $A$, Voltage response and corresponding spike raster plots to six stimulus presentations from a typical recording. $B-E$, Same analyses as shown in Figures 3 and 4 , but for the stimulus set using synchronous AMPA inputs in the absence of NMDA and synchronous $\mathrm{GABA}_{A}$ inputs $(n=9$ neurons recorded).

First, we compared how synchronized excitatory and inhibitory inputs compete in the control of spiking when only AMPA conductance was present in the excitation $\left(A_{\mathrm{s}}\right)$. In contrast to the condition of mixed AMPA and NMDA components, excitation now dominated in the generation of action potentials (Fig. 5). The spike pattern was highly similar for responses to stimuli with synchronous excitation in the presence or absence of synchronous inhibition $\left(A_{\mathrm{s}} G_{\mathrm{s}}\right.$ vs $A_{\mathrm{s}} G_{\mathrm{c}}$ ) but was quite different when the synchronous excitation was removed $\left(A_{\mathrm{s}} G_{\mathrm{s}}\right.$ vs $\left.A_{\mathrm{c}} G_{\mathrm{s}}\right)$. The dominant effect of excitation was confirmed by the comparison of spike timing precision within and across conditions (Fig. $5 C$ ). The impact of synchronized excitation was only slightly reduced by synchronized inhibition when compared with constant inhibition. In contrast, the impact of synchronized inhibition was strongly reduced by synchronized excitation (Fig. 5C). Furthermore, the STA of $G_{\text {ex }}$ was almost identical in the absence or presence of synchronized inhibition (Fig. 5D), and the peak amplitude of the STA of $G_{\text {in }}$ was strongly reduced compared with the case of mixed AMPA-NMDA excitation (Fig. 3E). These effects are likely because of the much-enhanced amplitude of transients in the $G_{\text {ex }}$ waveform in the absence of NMDA conductance (Fig.

\section{A: Spike Raster and Voltage traces}
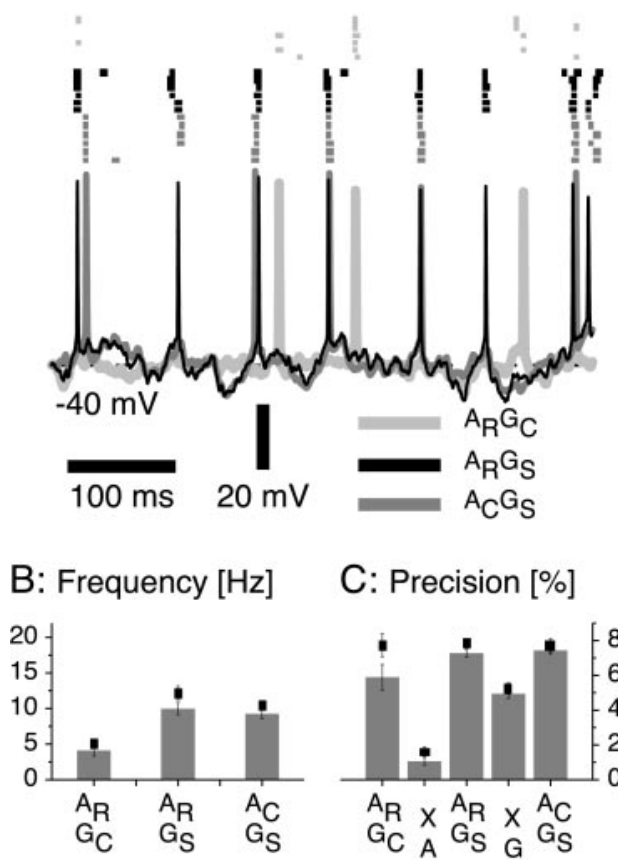

C: Precision [\%]
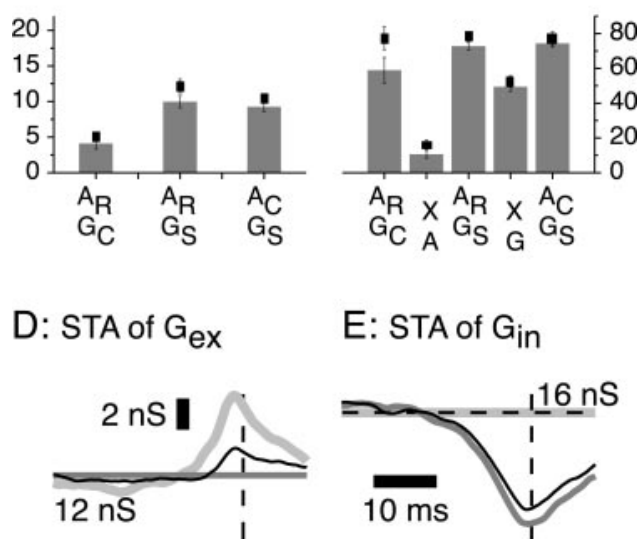

$E:$ STA of $G_{\text {in }}$

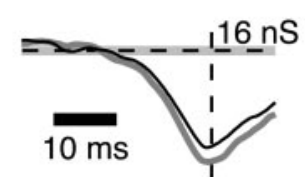

Figure 6. Control of DCN spiking by a combination of random pure AMPA input and synchronized $G_{A B A_{A}}$ inputs. $A_{R} G_{C}$ combines random excitatory AMPA input with constant inhibitory input (light gray). $A_{C} G_{S}$ combines constant excitatory input with synchronized inhibitory $\mathrm{GABA}_{\mathrm{A}}$-type input (dark gray). $A_{\mathrm{R}} G_{\mathrm{S}}$ combines random AMPA input with synchronized $\mathrm{GABA}_{\mathrm{A}}$ input (black). $A$, Voltage response and corresponding spike raster plots to six stimulus presentations from one DCN neuron. $B-E$, Same analyses as shown in Figure 5 , but for the stimulus set including random instead of synchronized AMPA inputs ( $n=9$ neurons recorded).

$1 C)$, which also is likely to account for the overall increase in spike rate, even though the average level of conductances remained identical.

To test whether AMPA excitation would override inhibitory input patterns even when excitatory inputs were not synchronized, we constructed the fourth stimulus set, comparing random AMPA input $\left(A_{\mathrm{r}}\right)$ and synchronized GABA input $\left(G_{\mathrm{s}}\right)$. This manipulation overall reduced the spike rate considerably (from $12 \mathrm{~Hz}$ for $A_{\mathrm{s}} G_{\mathrm{c}}$ to $4 \mathrm{~Hz}$ for $A_{\mathrm{r}} G_{\mathrm{c}}$ ), further indicating that transients caused by input synchronization were required for spike generation, even though the subthreshold membrane potential was very close to the spike threshold, and DCN neurons are endowed with persistent inward currents (Raman et al., 2000). In addition, the spike pattern was very similar between the conditions of random or constant excitation in the presence of synchronized inhibition (Fig. 6). Therefore, the spike generation was now mostly controlled by the $\mathrm{GABA}_{\mathrm{A}}$ input, and the roles between excitation and inhibition were reversed compared with the third stimulus set using synchronized excitation. The dominant influence of the $\mathrm{GABA}_{\mathrm{A}}$ input was confirmed by the corresponding spike timing precision values (Fig. 6C) and the STAs (Fig. $6 D, E$ ). The STA of $G_{\mathrm{s}}$ (Fig. $6 E$ ) was only slightly changed by switching $A_{\mathrm{r}}$ to $A_{\mathrm{c}}$, but the STA of $A_{\mathrm{r}}$ (Fig. 6D) was strongly 
reduced by switching from $G_{\mathrm{c}}$ to $G_{\mathrm{s}}$. Overall, these results confirm that the presence of a strong NMDA conductance in DCN neurons allows the inhibitory input of Purkinje cells to attain a more dominant role in the control of DCN spiking even when the excitation is strongly modulated by synchronous inputs. In contrast, an AMPA-only excitatory input dominates over inhibition, unless the temporal fluctuations in the inhibitory input significantly exceed those of the excitation.

\section{The role of the NMDA voltage dependence in synaptic integration}

Beyond the slower time course of NMDA compared with AMPA conductances that can account for the de-emphasis of excitatory input transients as demonstrated above, a key feature of the NMDA conductance is its voltage dependence. Although DCN neurons show only a weakly voltage-dependent NMDA conductance in comparison with other cell types (Anchisi et al., 2001), the NMDA current is still much reduced when the membrane potential is hyperpolarized (Fig. 1). In the recordings presented so far, the NMDA component was always voltage-dependent. To examine the functional significance of this voltage dependence, we constructed additional stimulus sets, in which stimuli with and without voltage dependence in the NMDA conductance were compared. First, we used an excitatory conductance $\left(G_{\text {ex }}\right)$ that had a voltage-dependent or independent NMDA component in the presence of synchronized inhibition. To isolate the effects of the NMDA voltage dependence, we used an excitatory conductance that was constant at any given voltage, i.e., was not constructed by summing unitary physiological synaptic inputs (Fig. $1 A, C, D)$. Nevertheless the voltage dependence of its NMDA component gave rise to amplitude fluctuations of $G_{\mathrm{ex}}$ as soon as $V_{\mathrm{m}}$ changed. Therefore we refer to this input as "exclusively voltage-modulated" in the following. Using the same conductance trace but with a fixed $V_{\mathrm{m}}$ of $-40 \mathrm{mV}$ in Equation 4 (see Materials and Methods) resulted in the voltage-independent $G_{\text {ex }}$ with a constant conductance of $12 \mathrm{nS}$. Comparing the responses to both stimuli, we isolated the effect of the voltage dependence of the NMDA component. Based on the stimulus construction method to keep the average excitatory conductance at $12 \mathrm{nS}$ (see Materials and Methods), the exclusively voltage-modulated NMDA conductance was identical to the voltage-independent constant conductance at $-40 \mathrm{mV}$, whereas it was higher or lower for values of $V_{\mathrm{m}}$ more depolarized or more hyperpolarized than $-40 \mathrm{mV}$, respectively. The membrane potential was more hyperpolarized with the exclusively voltage-modulated NMDA conductance during periods of hyperpolarization (Fig. 7.1A). Because the excitatory conductance was constant over time for the voltage-independent NMDA input in this stimulus, all spikes were induced by temporary decreases in inhibition (Fig. 7.1 E,F, gray traces). This was also true for the voltage-dependent NMDA input, but in addition, the depolarization caused by decreases in inhibition led to an increase in NMDA conductance because of its voltage dependence (Fig. 7.1 E,F, black traces). This was accompanied by an increase in spiking during periods of depolarization compared with voltage-independent NMDA conductance (Fig. 7.1A). Thus, NMDA voltage dependence boosted spike initiation much like expected from a persistent depolarization-activated inward conductance. The overall spike rate was increased by the NMDA voltage dependence, and the spike timing precision was not changed (Fig. $7.1 \mathrm{~B}$ ). The effect of the NMDA voltage dependence was even more pronounced when the inhibitory inputs contained only smaller temporal transients (Fig. 7.1 E, 7.2 E, compare black and gray lines), i.e., all 100 inhibitory inputs were activated at random (Fig. 7.2). In this case the spike rate more than doubled from 1.7 to $5.6 \mathrm{~Hz}$ in the presence of exclusively voltage-modulated NMDA input compared with a constant conductance, and the precise spike timing that was lost for the constant conductance was recovered (Fig. 7.2B). Of course, the NMDA conductance is usually modulated by temporal patterns in the excitatory input, and therefore we also investigated the effect of voltage dependence in our synchronous excitatory input condition when the inhibition was constant (Fig. 7.3). This stimulus was again designed such that voltage-dependent and independent NMDA conductances resulted in the same conductance waveforms for the reference potential of $-40 \mathrm{mV}$. In the presence of synchronized excitatory input, loss of the NMDA voltage dependence led to a decrease in spike frequency as well as spike precision (compare Figs. 3, 7.3B). Again, NMDA voltage dependence was boosting the excitatory conductance preceding each spike (Fig. $7.3 F$ ) because of the increasing NMDA conductance as the cell depolarized toward spike threshold.

We further analyzed the data comparing voltage-dependent and voltage-independent NMDA conductances to elucidate the mechanisms underlying the response boosting by NMDA voltage dependence. From the stimulus construction alone, it was clear that a depolarization of more than $-40 \mathrm{mV}$ would lead to a larger NMDA conductance for the voltage-dependent forms. The functional significance for this depolarization-dependent increase in NMDA conductance is clear from the spike-triggered averages (Fig. 7.1C-F, 7.2C-F, 7.3C-F) because the presence of NMDA voltage dependence led to a larger increase of $G_{\mathrm{ex}}$ before spike threshold was reached (Fig. 7.1F,7.2F,7.3F). Accordingly, extra spikes were generated on average by an additional membrane depolarization of $\sim 4 \mathrm{mV}$ that started to develop only $\sim 5-10$ msec before spike generation (Fig. 7.1 D,7.2D,7.3D, thin black traces). Therefore, less pronounced depolarizing inputs (Fig. 7.1 E, 7.2 E, dotted lines, 7.3F, gray line) were enabled to trigger action potentials by the voltage dependence of the NMDA input. Spikes that were already triggered by input transients in the voltage-independent NMDA condition generally were triggered 1-2 msec earlier in the voltage-dependent condition, as demonstrated by a cross-correlation analysis (data not shown). These findings were common regardless of whether temporal fluctuations of inhibition or excitation dominated, indicating that a common role of NMDA voltage dependence consists of boosting the spike response to input conditions that cause depolarization. Given the DCN circuitry, this signifies that the effect of pauses in Purkinje cell spiking is enhanced by the presence of a baseline of NMDA conductance because of ongoing excitatory input from mossy and climbing fiber collaterals. A second possible contribution to response boosting by NMDA voltage dependence could originate from a deinactivation of sodium channels during periods of hyperpolarization preceding spiking, which were enhanced in the presence of NMDA voltage dependence. The membrane potential at which spikes were triggered was not lowered in the presence of voltage-dependent NMDA conductance, however, as shown by the identical inflection points of STA waveforms of $V_{\mathrm{m}}$ in Figures 7.1C, 7.2C, and 7.3C. Thus, the spike threshold remained constant, which could be caused by an insufficient amount of additional hyperpolarization with voltagedependent NMDA conductance to increase Na channel deinactivation or to a compensatory deinactivation of $\mathrm{K}$ channels.

\section{Discussion}

Purkinje cells and mossy fibers show ongoing activity in vivo (Eccles et al., 1971; Cazin et al., 1980; Savio and Tempia, 1985; 


\section{1.: $\mathrm{AN}_{\mathrm{C}} \mathrm{G}_{\mathrm{S}}$}

$7.1 \mathrm{~A}$

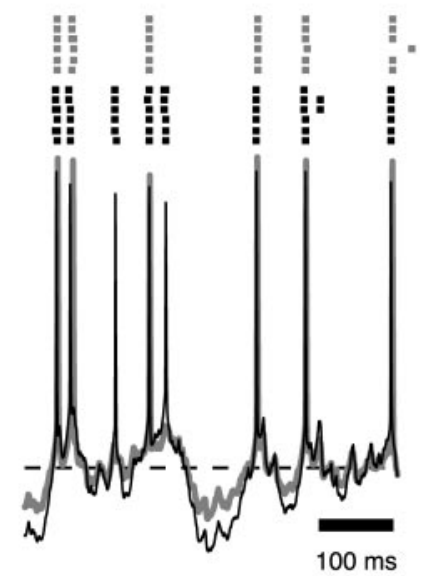

7.1B
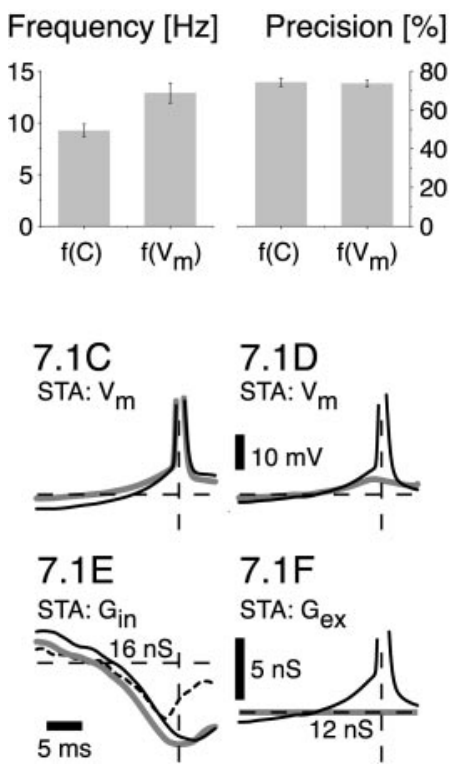

\section{2.: $\mathrm{AN}_{\mathrm{C}} \mathrm{G}_{\mathrm{R}}$}

$7.2 \mathrm{~A}$

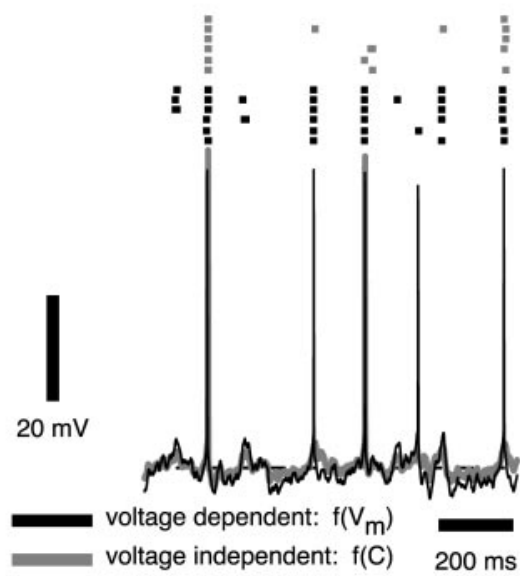

7.2B
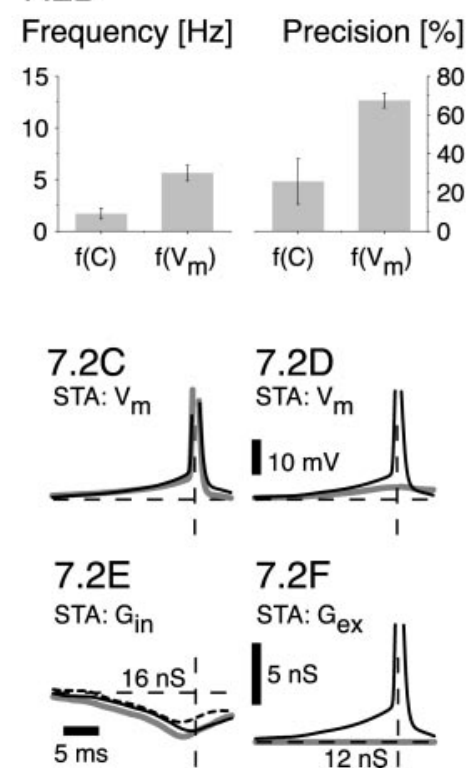

\section{3.: $\mathrm{ANS}_{\mathrm{S}}$}

7.3A

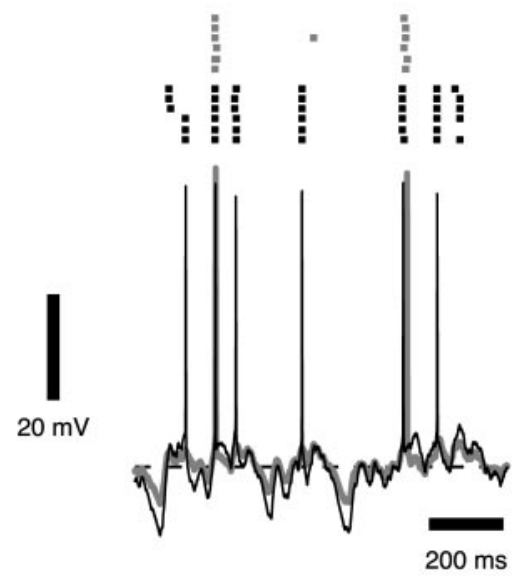

7.3B
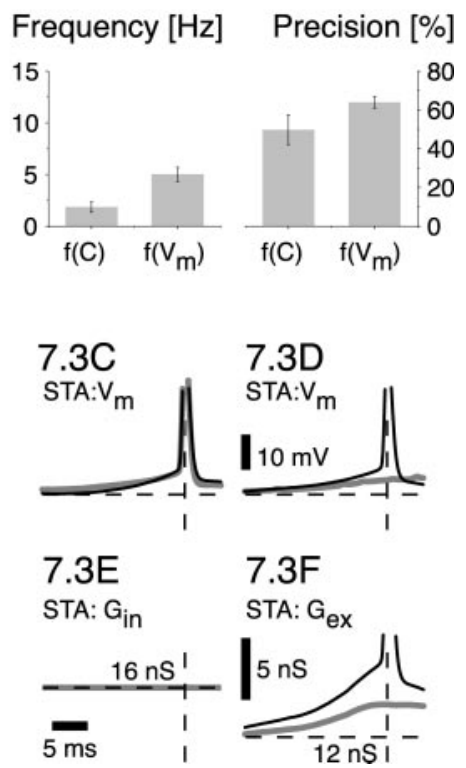

Figure 7. Effect of NMDA voltage dependence on spike rate and spike timing precision. 7.1, Comparison of voltage-independent and exclusively voltage-modulated excitatory input in the presence of synchronous inhibitory inputs ( $n=25$ neurons). 7.2, Comparison of voltage-independent and exclusively voltage-modulated excitatory input in the presence of random inhibitory inputs ( $n=10$ neurons). 7.3, Comparison of voltage-dependent and independent synchronous excitatory input in the presence of constant inhibitory inputs ( $n=9$ neurons). The analysis is identical to that used in previous figures, except that STAs of the membrane potential have been added in subpanels $C$ and $D$. In subpanels $C$, the analysis was restricted to spikes that occurred both in the presence and absence of NMDA voltage dependence, whereas in subpanels $D$, the STAs were calculated from spikes that occurred only in the presence of NMDA voltage dependence. The black lines in D show the STAs for the voltage-dependent input, whereas the gray lines show the cross-stimulus STAs for the voltage-independent input. Because the temporal pattern of inputs was identical in both conditions, the cross-stimulus STA shows the subthreshold trajectory for input events that triggered spikes in the voltage-dependent but not the voltage-independent stimulus. The STAs of $G_{\text {in }}$ shown in subpanels $E$ were calculated from the same spikes as the STAs in subpanels C (solid lines) and from the same spikes as the STAs in subpanels $D$ (dotted lines). The STAs of $G_{\text {ex }}$ shown in subpanels $F$ were calculated from the same spikes as the STAs in subpanels $D$ (solid lines). The calculation of STAs was restricted to spikes with a minimum preceding ISI of 50 msec to avoid contamination of the prespike depolarization with preceding spikes in single trials.

Stratton et al., 1988; van Kan et al., 1993; Gamlin and Clarke, 1995; Matsuzaki and Kyuhou, 1997). To examine the control of DCN spiking by such an input condition, we used dynamic clamping in vitro to allow complete control over complex input patterns. Based on our previous findings that temporal modulation of inhibitory inputs is very important in triggering spiking (Gauck and Jaeger, 2000), we examined how temporal modulation of excitation and inhibition may interact. We found that because of the large and slow NMDA conductance in the excitatory input of DCN neurons (Anchisi et al., 2001), inhibitory input patterns dominated in the control of spike timing. This condition could be reversed, when the temporal modulation in the inhibitory pathway was much less pronounced than that in the excitation. In addition, we found that the voltage dependence of NMDA conductance played a significant role in enhancing spike responses to depolarizing input transients. Such transients were caused either by transient reductions in inhibitory input or by increases in excitatory input.

One important question is whether our findings are relevant for all likely activity patterns in vivo and are not restricted to our somewhat arbitrary manipulation of input patterns consisting of switching between random input and completely synchronized sets of input fibers. We believe that this is the case for the following reasons. First, the range of input conductance waveforms that 
we tested by using different synchronization levels (Fig. 1) contained a broad spectrum of time courses and amplitude transients that likely encompass many conductance waveforms that would also result from other input conditions. Second, the use of highly synchronized input conditions is indicated by a number of publications that point toward possible mechanisms (see Functional considerations below) for the synchronization of Purkinje cell activity (Bower and Woolston, 1983; Pichitpornchai et al., 1994; Cohen and Yarom, 1998; Gundappa-Sulur et al., 1999; Lang et al., 1999; Vos et al., 1999; Mann-Metzer and Yarom, 1999, 2000). Third, we prefer our construction of input patterns based on summing unitary EPSCs and IPSCs to subjecting neurons to white noise stimuli, because white noise will contain many features not attainable by synaptic conductances and will underrepresent the most common conductance patterns following synaptic inputs. To restrict the input conditions to a manageable number, we decided to omit any specific correlations between excitatory and inhibitory inputs. The space of possible such correlations is extremely large and is ill constrained by experimental data. In the many random conditions of combined excitatory and inhibitory transients in our inputs, we found no evidence for very specific conjunctions needed to control spiking. Rather, spikes were favored generally by any combination of reduced inhibition and increased excitation. For both input streams, individual spikes generally showed a dependence on the preceding waveforms for 10-20 msec. This specific temporal window of synaptic integration is likely shaped by the active properties of DCN neurons. A recent publication indicates for example that the Hodgkin-Huxley properties of sodium channels might be sufficient to shift the sensitivity of pyramidal neurons dynamically from slower to faster depolarizing input transients depending on the level of input activity (Azouz and Gray, 2003). The temporal window of synaptic integration preceding spike generation is overall likely to be caused by the precise kinetics of all voltagegated channels present. A pharmacological dissection of the involvement of particular ionic currents was beyond the scope of the present study, however.

\section{The response to cerebellar cortical input in the DCN}

Based on our results, we propose that DCN spiking is controlled in two ways. First, the relative balance of excitation and inhibition over an extended period of time is important in setting up an average spike frequency (Gauck and Jaeger, 2000). Second, temporal transients in the inputs caused by presynaptic synchronization in activity are very important in allowing spikes to be triggered at specific times. In fact, when input conductances are completely constant, even a strong excitatory baseline is unlikely to trigger spikes because of the voltage-clamping effect exerted by synaptic conductances (Gauck and Jaeger, 2000), casting doubt on the relevance of pure rate coding for DCN neurons. In the present study we find that DCN neurons are selective to respond strongly to transients in inhibitory inputs, because excitatory transients are dampened by slow NMDA conductances. The most effective inhibitory transients were brief pauses in inhibitory input, which caused immediate spike responses, as seen by the method of spike-triggered averaging. Spike-triggered averages showed no evidence of spikes after pronounced increases of inhibition, as would be the case with post-hyperpolarization rebound spiking. Rebound spiking, which has been observed in DCN neurons after strong inhibitory transients in vitro in the absence of ongoing excitation (Aizenman and Linden, 1999), thus played no role in input processing under our input conditions of a continuous balance of excitation and inhibition. Con- cepts about synchronization-based information processing have been suggested for the cerebral cortex (Abeles, 1991; Diesmann et al., 1999; Singer, 1999; Gray, 1999), but have focused on excitatory input transients. The basic idea is that cerebral cortical neurons respond selectively to incoming pulse packets of synchronized excitation. The present study indicates that such a mechanism would not necessarily be limited to excitatory inputs, because reductions in inhibitory input create similar input transients in the presence of an excitatory conductance baseline. Interestingly, a depolarization caused by a reduction in conductance is somewhat better suited to trigger a spike than the same depolarization triggered by excitation, because the shunting force of the input conductance is reduced, allowing an easier spike escape caused by voltage-gated currents. Nevertheless, we found that strong excitatory transients could reliably trigger spikes in DCN neurons, even though the excitatory conductance was smoothed out by the large NMDA component.

\section{The role of the NMDA voltage dependence}

The voltage-dependent magnesium block of the NMDA input to DCN neurons is rather weak compared with that found in many other neuron types (Kuner and Schoepfer, 1996), suggesting that it might be of secondary functional significance. To study the contribution of the voltage dependence of the NMDA input to the activity control of DCN neurons, we compared their responses to voltage-dependent and voltage-independent stimuli. The results showed that the spike frequency as well as the spike timing precision were increased by the voltage dependence of the NMDA input. An increase in the spike timing precision was especially large for inhibitory input with a low level of synchronization. This indicates that the presence of the large voltagedependent NMDA component facilitates the impact that $\mathrm{GABA}_{\mathrm{A}}$ type input can exert on the activity of DCN neurons. Furthermore, the timing of spikes triggered by transient reductions in inhibitory input was almost identical with voltage-independent or voltage-dependent NMDA input, indicating that NMDA enhancement of responses did not impair the fidelity of these responses. Overall, the large weakly voltage-dependent NMDA component of the excitatory input conductance in DCN neurons is thus well suited to enhance DCN spiking caused by decreases in inhibition but not to override the effect of increases in inhibitory input. Although we did not directly test the effect that strongly voltage-dependent NMDA conductances would have in this situation, it seems likely that because of the large NMDA input component, this could lead to a full bistability of DCN neurons and NMDA-induced bursting independent of inhibitory inputs. It is possible that the weak voltage dependence developed to disallow such escapes from cerebellar cortical inhibitory input.

\section{Functional considerations}

Taken together our data indicate that excitatory as well as inhibitory inputs can determine the spiking activity of DCN neurons, depending on the presence and strength of rapid fluctuations in the respective input pathway. Given similar levels of fluctuations in both input pathways, the inhibition had privileged control over spike initiation, however. Because of the high convergence of many Purkinje cells onto a single DCN neuron (Palkovits et al., 1977), a population of Purkinje cells is likely required to show correlated transients in activity for DCN neurons to respond. There are several lines of evidence supporting the notion that populations of Purkinje cells might contain strong correlated transients in activity. First, climbing fiber inputs can be synchronized among large numbers of Purkinje cells, and in turn syn- 
chronize Purkinje cell complex spiking (Lang et al., 1999). Mossy fiber input could synchronize Purkinje cell activity via their highly branched projection to multiple areas of granule cells and activating ascending granule cell axons, which have a strong influence on overlying Purkinje cells (Bower and Woolston, 1983; Pichitpornchai et al., 1994; Cohen and Yarom, 1998; GundappaSulur et al., 1999). Furthermore, not only excitatory but also inhibitory input could synchronize the activity between Purkinje cells (Jaeger et al., 1997; Jaeger and Bower, 1999). In this respect it is interesting to note that the activity of inhibitory interneurons of the cerebellar cortex has been reported to be synchronized by parallel fiber activity (Vos et al., 1999) and gap junctions (MannMetzer and Yarom, 1999, 2000). The possibility that strong sensory inputs via the mossy fiber and climbing fiber systems may trigger direct excitatory responses in the DCN is also supported by the present study. Our results suggest that it would be particularly fruitful to monitor large numbers of Purkinje cells and DCN neurons in awake behaving animals with multiwire designs to further elucidate network activity during the control of behavior.

\section{References}

Abeles M (1991) Corticonics. Cambridge, MA: Cambridge UP.

Aizenman CD, Linden DJ (1999) Regulation of the rebound depolarization and spontaneous firing patterns of deep nuclear neurons in slices of rat cerebellum. J Neurophysiol 82:1697-1709.

Anchisi D, Scelfo B, Strata P, Tempia F (1998) Postsynaptic currents in deep cerebellar nuclei. Soc Neurosci Abstr 24:325.

Anchisi D, Scelfo B, Tempia F (2001) Postsynaptic currents in deep cerebellar nuclei. J Neurophysiol 85:323-331.

Audinat E, Gahwiler BH, Knopfel T (1992) Excitatory synaptic potentials in neurons of the deep nuclei in olivo-cerebellar slice cultures. Neuroscience 49:903-911.

Azouz R, Gray CM (2003) Adaptive coincidence detection and dynamic gain control in visual cortical neurons in vivo. Neuron 37:513-523.

Batini C, Compoint C, Buisseret-Delmas C, Daniel H, Guegan M (1992) Cerebellar nuclei and the nucleocortical projections in the rat: retrograde tracing coupled to GABA and glutamate immunohistochemistry. J Comp Neurol 315:74-84.

Bower JM, Woolston DC (1983) Congruence of spatial organization of tactile projections to granule cell and Purkinje cell layers of cerebellar hemispheres of the albino rat: vertical organization of cerebellar cortex. J Neurophysiol 49:745-766.

Cazin L, Precht W, Lannou J (1980) Firing characteristics of neurons mediating optokinetic responses to rat's vestibular neurons. Pflügers Arch 386:221-230

Cohen D, Yarom Y (1998) Patches of synchronized activity in the cerebellar cortex evoked by mossy-fiber stimulation: questioning the role of parallel fibers. Proc Natl Acad Sci USA 95:15032-15036.

Cull-Candy SG, Brickley SG, Misra C, Feldmeyer D, Momiyama A, Farrant M (1998) NMDA receptor diversity in the cerebellum: identification of subunits contribution to functional receptors. Neuropharmacology 37:1369-1380.

De Zeeuw CI, Berrebi AS (1995) Postsynaptic targets of Purkinje cell terminals in the cerebellar and vestibular nuclei of the rat. Eur J Neurosci 7:2322-2333.

Diesmann M, Gewaltig MO, Aertsen A (1999) Stable propagation of synchronous spiking in cortical neural networks. Nature 402:529-533.

Eccles JC, Faber DS, Murphy JT, Sabah NH, Taborikova H (1971) Afferent volleys in limb nerves influencing impulse discharges in cerebellar cortex. I. In mossy fibers and granule cells. Exp Brain Res 13:15-35.

Eccles JC, Sabah NH, Schmidt RF, Taborikova H (1972) Cutaneous mechanoreceptors influencing impulse discharges in cerebellar cortex. II. In Purkinje cells by mossy fiber input. Exp Brain Res 15:261-277.

Gamlin PD, Clarke RJ (1995) Single-unit activity in the primate nucleus reticularis tegmenti pontis related to vergence and ocular accommodation. J Neurophysiol 73:2115-2119.

Gauck V, Jaeger D (2000) The control of rate and timing of spikes in the deep cerebellar nuclei by inhibition. J Neurosci 20:3006-3016.
Gray CM (1999) The temporal correlation hypothesis of visual feature integration: still alive and well. Neuron 24:31-47.

Gundappa-Sulur G, De Schutter E, Bower JM (1999) Ascending granule cell axon: an important component of cerebellar cortical circuitry. J Comp Neurol 408:580-596.

Harsch A, Robinson HP (2000) Postsynaptic variability of firing in rat cortical neurons: the roles of input synchronization and synaptic NMDA receptor conductance. J Neurosci 20:6181-6192.

Jaeger D, Bower JM (1999) Synaptic control of spiking in cerebellar Purkinje cells: dynamic current clamp based on model conductances. J Neurosci 19:12.

Jaeger D, De Schutter E, Bower JM (1997) The role of synaptic and voltagegated currents in the control of Purkinje cell spiking: a modeling study. J Neurosci 17:91-106.

Kuner T, Schoepfer R (1996) Multiple structural elements determine subunit specificity of $\mathrm{Mg}^{2+}$ block in NMDA receptor channels. J Neurosci 16:3549-3558.

Lang EJ, Sugihara I, Welsh JP, Llinas R (1999) Patterns of spontaneous Purkinje cell complex spike activity in the awake rat. J Neurosci 19:2728-2739.

Mann-Metzer P, Yarom Y (1999) Electrotonic coupling interacts with intrinsic properties to generate synchronized activity in cerebellar networks of inhibitory interneurons. J Neurosci 19:3298-3306.

Mann-Metzer P, Yarom Y (2000) Electrotonic coupling synchronizes interneuron activity in the cerebellar cortex. Prog Brain Res 124:115-122.

Matsuzaki R, Kyuhou S (1997) Pontine neurons which relay projections from the superior colliculus to the posterior vermis of the cerebellum in the cat: distribution and visual properties. Neurosci Lett 236:99-102.

Palkovits M, Mezey E, Hamori J, Szentagothai J (1977) Quantitative histological analysis of the cerebellar nuclei in the cat. I. Numerical data on cells and synapses. Exp Brain Res 28:189-209.

Pedroarena CM, Schwarz C (2003) Efficacy and short-term plasticity at GABAergic synapses between Purkinje cells and cerebellar nuclei neurons. J Neurophysiol 89:704-715.

Pichitpornchai C, Rawson JA, Rees S (1994) Morphology of parallel fibres in the cerebellar cortex of the rat: an experimental light and electron microscopic study with biocytin. J Comp Neurol 342:206-220.

Raman IM, Gustafson AE, Padgett D (2000) Ionic currents and spontaneous firing in neurons isolated from the cerebella nuclei. J Neurosci 20:9004-9016.

Robinson HP, Kawai N (1993) Injection of digitally synthesized synaptic conductance transients to measure the integrative properties of neurons. J Neurosci Methods 49:157-165.

Savio T, Tempia F (1985) On the Purkinje cell activity increase induced by suppression of inferior olive activity. Exp Brain Res 57:456-463.

Sharp AA, O'Neil MB, Abbott LF, Marder E (1993) Dynamic clamp: computer-generated conductances in real neurons. J Neurophysiol 69:992-995.

Shinoda Y, Sugihara I, Wu HS, Sugiuchi Y (2000) The entire trajectory of single climbing and mossy fibers in the cerebellar nuclei and cortex. Prog Brain Res 124:173-186.

Singer W (1999) Time as coding space? Curr Opin Neurobiol 9:189-194.

Stratton SE, Lorden JF, Mays LE, Oltmans GA (1988) Spontaneous and harmaline-stimulated Purkinje cell activity in rats with a genetic movement disorder. J Neurosci 8:3327-3336.

Sugihara I, Wu H, Shinoda Y (1999) Morphology of single olivocerebellar axons labeled with biotinylated dextran amine in the rat. J Comp Neurol 414:131-148.

Sultan F, Czubayko U, Thier P (2003) Morphological classification of the rat lateral cerebellar nuclear neurons by principal component analysis. J Comp Neurol 455:139-155.

Telgkamp P, Raman IM (2002) Depression of inhibitory synaptic transmission between Purkinje cells and neurons of the cerebellar nuclei. J Neurosci 22:8447-8457.

van Kan PL, Gibson AR, Houk JC (1993) Movement-related inputs to intermediate cerebellum of the monkey. J Neurophysiol 69:74-94.

Vos BP, Maex R, Volny-Luraghi A, De Schutter E (1999) Parallel fibers synchronize spontaneous activity in cerebellar Golgi cells. J Neurosci 19:RC6.

Wu H-S, Sugihara I, Shinoda Y (1999) Projection patterns of single mossy fibers originating from the lateral reticular nucleus in the rat cerebellar cortex and nuclei. J Comp Neurol 411:97-118. 